\title{
Fratura de Agulha durante Punção Subaracnóidea. Relato de Caso *
}

\section{Needle Fracture during Spinal Puncture. Case Report}

\author{
Marcos G C Cruvinel, TSA ${ }^{1}$; André V C Andrade ${ }^{2}$
}

\author{
RESUMO \\ Cruvinel MGC, Andrade AVC - Fratura de Agulha durante Pun- \\ ção Subaracnóidea. Relato de Caso
}

\begin{abstract}
JUSTIFICATIVA E OBJETIVOS: Depois da introdução das agulhas de fino calibre (26G, 27G e 29G) e a conseqüente diminuição da incidência de cefaléia pós-punção da dura-máter, a raquianestesia vem sendo cada dia mais empregada. Suas complicações são pouco freqüentes. Recentemente, entretanto, foi observada uma complicação aparentemente rara, de fratura da agulha durante punção subaracnóidea. O objetivo deste relato é registrar o fato e aventar as possíveis causas e sua prevenção.
\end{abstract}

RELATO DO CASO: Paciente do sexo masculino, 53 anos, 90 $\mathrm{kg}, 175 \mathrm{~cm}$, portador de hipertensão arterial sistêmica crônica e insuficiência renal crônica apresentou-se, em caráter de urgência, para transplante renal. Foi proposta a administração de morfina subaracnóidea visando analgesia pós-operatória. Inicialmente tentou-se, sem sucesso, punção em $L_{3}-L_{4}$ com agulha 27G, seguiu-se tentativa de punção em $L_{2}-L_{3}$ quando se percebeu deformação da agulha. Ao ser retirada a agulha partiu-se, permanecendo um fragmento de $43 \mathrm{~mm}$ no ligamento interespinhoso de $L_{2}-L_{3}$, confirmado por fluoroscopia. Foi induzida anestesia geral e procedeu-se exploração cirúrgica guiada por fluoroscopia com retirada do fragmento da agulha. A seguir, o transplante renal foi realizado sem intercorrências.

CONCLUSÕES: A diminuição do calibre das agulhas, que trouxe a vantagem da redução da incidência de cefaléia pós-punção da dura-máter, fez também com que elas se tornassem mais frágeis. Em casos onde os ligamentos são mais resistentes e a punção tentada mais de uma vez, pode haver deformação do material que fica susceptível a quebra. Conclui-se que em situações em que há resistência aumentada à passagem da agulha ou dificuldade de punção, o risco de sua deformação e a possibilidade de fratura devem ser confrontados com as vantagens do uso de agulhas de fino calibre.

Unitermos: COMPLICAÇÕES; TÉCNICAS ANESTÉSICAS, Regional: subaracnóidea

\footnotetext{
* Recebido do (Received from) Departamento de Anestesiologia do Hospital Vera Cruz, Belo Horizonte, MG

1. Anestesiologista dos Hospitais Vera Cruz e Life Center

2. Anestesiologista do Hospital Vera Cruz
}

Apresentado (Submitted) em 08 de abril de 2004

Aceito (Accepted) para publicação em 12 de agosto de 2004.

Endereço para correspondência (Correspondence to)

Dr. Marcos Guilherme Cunha Cruvinel

Rua Simão Irffi, 86/301

30380-270 Belo Horizonte, MG

(C) Sociedade Brasileira de Anestesiologia, 2004

\author{
SUMMARY \\ Cruvinel MGC, Andrade AVC - Needle Fracture during Spinal \\ Puncture. Case Report
}

BACKGROUND AND OBJECTIVES: After fine spinal needles (26G, $27 \mathrm{G}$ and 29G) introduction and consequent decrease in post dural puncture headache, spinal anesthesia is being increasingly used. Its complications are uncommon however recently we have observed a seemingly unusual complication: needle fracture during spinal puncture. This report aimed at describing the fact and at pointing its possible causes in addition to its prevention.

CASE REPORT: Male patient, 53 years old, $90 \mathrm{~kg}, 175 \mathrm{~cm}$, with chronic systemic hypertension and renal failure presented for emergency renal transplantation. Spinal morphine was proposed for postoperative analgesia. Puncture at $L_{3}-L_{4}$ with $27 \mathrm{G}$ needle was attempted without success, and was followed by attempt at $L_{2}-L_{3}$ when needle deformation was observed. Needle has broken at removal, leaving behind a $43 \mathrm{~mm}$ fragment in the $L_{2}-L_{3}$ interspinous ligament, which was confirmed by fluoroscopy. General anesthesia was induced and fragment was removed by fluoroscopic surgical exploration. Renal transplantation was then performed uneventfully.

CONCLUSIONS: Decreased needle gauge has decreased post dural puncture headache but has also made needles fragiles. When ligaments are more resistant and puncture is attempted more than once there may be material stress leading to needle fracture. In conclusion, when there is increased resistance to needle introduction and puncture difficulty, the risk of deformation and potential fracture should be balanced against fine needle advantages.

Key Words: ANESTHETIC TECHNIQUES, Regional: spinal block; COMPLICATIONS

\section{INTRODUÇÃO}

raquianestesia é técnica de execução simples e eficienAte. Além de praticamente ausente o risco de toxicidade sistêmica pelo anestésico local, tem rápido início de ação, promove analgesia completa com ótimo relaxamento muscular, sendo técnica muito empregada em nosso meio. Com a introdução das agulhas de fino calibre (26G, 27G e 29G) e a conseqüente diminuição da incidência de cefaléia pós-punção de dura-máter, a raquianestesia é, provavelmente, a técnica de anestesia regional mais empregada nos dias de hoje ${ }^{1-3}$. É considerada como técnica de escolha em grande número de situações, sendo empregada rotineiramente com baixa incidência de complicações. A complicação mais citada na literatura é a cefaléia pós-punção da dura-máter. Outras complicações menos freqüentes são faIha, dor lombar, bradicardia, bloqueio alto, complicação neurológica, como síndrome da cauda eqüina, sintomas neurológicas temporários, lesões neurológicas permanentes incluindo a paraplegia, parada cardíaca e morte ${ }^{3-13}$. Recentemente, entretanto, constatou-se uma complicação rara, pouco citada na literatura, de fratura de agulha durante 
punção subaracnóidea ${ }^{14,15}$. O objetivo deste relato é registrar o fato e aventar as possíveis causas e sua prevenção.

\section{RELATO DO CASO}

Paciente do sexo masculino, 53 anos, $90 \mathrm{~kg}, 175 \mathrm{~cm}$, portador de hipertensão arterial sistêmica crônica e insuficiência renal crônica em conseqüência de nefropatia hipertensiva apresentou-se, em caráter de urgência, para transplante renal. Encontrava-se em programa de hemodiálise com exames pré-operatórios sem alterações significativas exceto por aumento da creatinina e da uréia, acidose metabólica compensada, discreta anemia e disfunção diastólica ao ecocardiograma. Foi proposta a administração de $200 \mu \mathrm{g}$ de morfina, por via subaracnóidea, visando analgesia pós-operatória. Após monitorização com eletrocardiograma, oxímetro de pulso e pressão arterial média contínua, o paciente foi sedado com $3 \mathrm{mg}$ de midazolam e colocado na posição sentada para a realização do bloqueio. Não houve dificuldade de localização dos espaços entre os processos espinhosos de $\mathrm{L}_{2}-\mathrm{L}_{3}$ e $\mathrm{L}_{3}-\mathrm{L}_{4}$. Inicialmente tentou-se, sem sucesso, punção em $L_{3}-L_{4}$ com agulha $27 \mathrm{G}$ Quincke $\left(B\right.$ Braun ${ }^{\circledR}$ ). Seguiu-se nova tentativa de punção em $L_{2}-L_{3}$ quando se percebeu deformação da agulha. A resistência à retirada do mandril estava aumentada e ele se encontrava discretamente deformado (Figura 1). Como não houve refluxo de líquor, confirmou-se a suspeita de que a possível progressão da agulha se deu devido à sua deformação e não a seu avanço para o espaço subaracnóideo. Ao ser retirada, a agulha partiu-se, permanecendo um fragmento de $43 \mathrm{~mm}$ (Figura 2) no ligamento interespinhoso de $L_{2}-L_{3}$, confirmado por fluoroscopia.

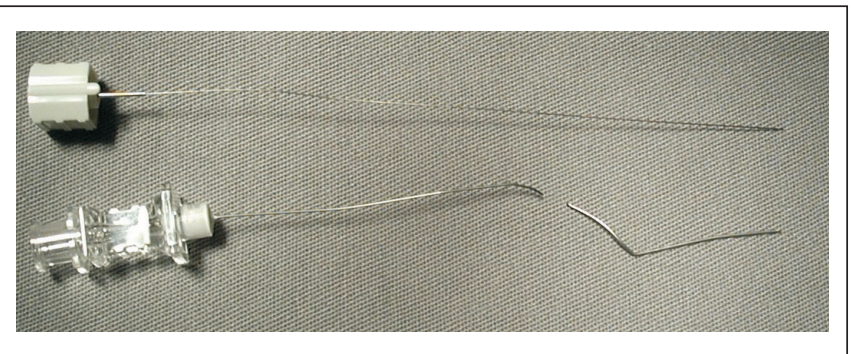

Figura 1 - Agulha de Raquianestesia 27G Quincke (B Braun $\left.{ }^{8}\right)$ Fraturada e seu Respectivo Mandril Deformado

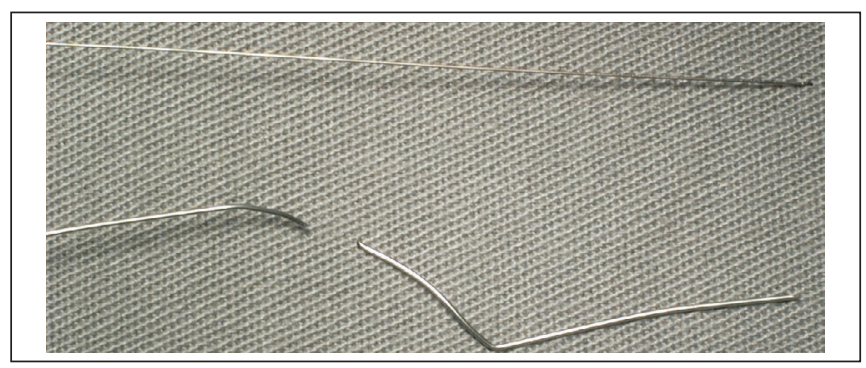

Figura 2 - Detalhe da Agulha de Raquianestesia 27G Quincke (B Braun $\left({ }^{\circledR}\right)$ Fraturada a $43 \mathrm{~mm}$ de sua Ponta e seu Mandril. Fragmento Distal Retirado após Exploração Cirúrgica da Região do Ligamento Interespinhoso de $\mathrm{L}_{2}-\mathrm{L}_{3}$
Afigura 3 mostra o provável mecanismo de sua deformaçãoe ruptura. Após indução de anestesia geral, procedeu-se a exploração cirúrgica guiada por fluoroscopia com retirada do fragmento da agulha. Aseguir, o transplante renal foi realizado sem intercorrências.

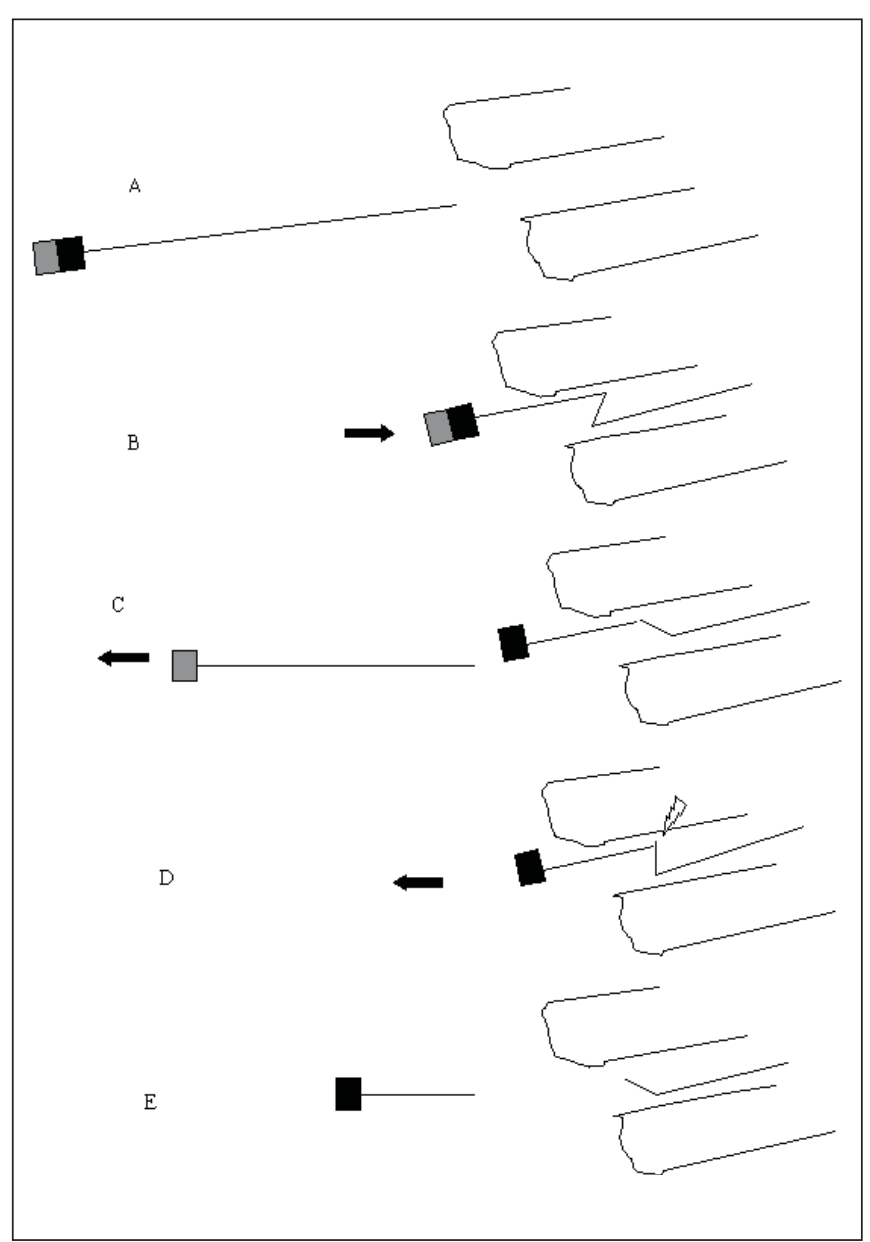

Figura 3 - Desenho Mostrando o Provável Mecanismo de Deformação da Agulha (A, B e C) e de sua Fratura (D e E)

\section{DISCUSSÃo}

Embora seja rara e, em um primeiro momento tenha causado muita surpresa, uma reflexão mais cuidadosa mostrou que esta complicação não é totalmente inesperada. Benham ${ }^{16}$ examinou 222 agulhas encontrando deformação em 36 $(16,2 \%)$ delas. Entre os diferentes tipos e calibres de agulha, as $25 \mathrm{G}$ danificaram-se significativamente menos que as de menor calibre. A diminuição do calibre das agulhas, que trouxe a vantagem da redução da incidência de cefaléia pós-punção da dura-máter, fez também com que elas se tornassem mais frágeis, levando a maior dificuldade de punção e maior probabilidade de falhas ${ }^{3-9}$. Em casos onde os ligamentos são mais resistentes e múltiplas punções são realizadas, pode haver desgaste do material que fica susceptível à que- 
bra. Uma incidência de $7,29 \%$ de mais de quatro tentativas para bloqueios do neuro-eixo foi encontrada por Oliveira FiIho e col. ${ }^{17}$. Entre os quatro fatores preditores de dificuldade de punção estudados por este autor, o paciente do presente caso apresentava três. São eles; idade maior que 40 anos, índice de massa corporal maior que $25 \mathrm{~kg} \cdot \mathrm{m}^{-2}$ e ser brevilíneo. Ele não apresentava aparentemente alterações anatômicas da coluna.

Chaney ${ }^{18}$ chama a atenção que com a utilização de agulhas de pequeno calibre grandes deformidades da agulha são possíveis, a despeito da aparência externa de punção rotineira. Imbelloni e col. ${ }^{19}$ relataram deformidade com agulha $29 \mathrm{G} \mathrm{e}$ salientou que este fato nunca ocorreu com agulha $27 \mathrm{G}$, diferente do presente caso. Embora seja pouco freqüente, a deformação acentuada da agulha ocorre, eventualmente, em casos de punção difícil. Em todos eles a conduta é sua imediata substituição.

Aindicação de punção com agulha de fino calibre no paciente em questão, é discutível, uma vez que a cirurgia seria de grande porte, não se tratava de paciente jovem e tinha preditores de punção difícil. Talvez a utilização de agulha mais grossa, calibre 25G, não representasse um maior risco de cefaléia e teria menor probabilidade de deformação. Outro ponto a considerar é a insistência na punção com a agulha $27 \mathrm{G}$. Mesmo não havendo até aquele momento sinais visíveis de desgaste da agulha, após a primeira tentativa de punção mal sucedida, provavelmente a decisão mais acertada teria sido trocá-la.

Em conclusão, o presente caso sugere que, em situações em que há resistência aumentada à progressão da agulha ou dificuldade de punção, o risco de deformação e a conseqüente possibilidade de fratura devem ser avaliados em relação as vantagens do uso de agulhas de fino calibre.

\section{Needle Fracture during Spinal Puncture. Case Report}

\section{Marcos G C Cruvinel, TSA, M.D.; André V. C. Andrade, M.D.}

\section{INTRODUCTION}

Spinal anesthesia is simple and effective. In addition to virtually no systemic toxicity risk, it has fast onset, promotes total analgesia with excellent muscle relaxation and is widely used in our country. After the introduction of fine needles (26G, 27G and 29G) and consequent decrease in post dural puncture headache it has become the most widely used regional technique ${ }^{1-3}$. It is the procedure of choice in many situations, being routinely used with low incidence of complications. Most common complication in the literature is post dural puncture headache. Other less frequent complications include failure, low back pain, bradycardia, upper blockade, neurological complications such as cauda equina syndrome, transient neurological symptoms and permanent neurological injuries including paraplegia, cardiac arrest and death ${ }^{3-13}$. However, an uncommon complication seldom described in the literature has been recently observed, namely needle fracture during spinal puncture ${ }^{14,15}$. This report aimed at describing the fact and at pointing its possible causes in addition to its prevention.

\section{CASE REPORT}

Male patient, 53 years old, $90 \mathrm{~kg}, 175 \mathrm{~cm}$, with chronic systemic hypertension and renal failure following hypertensive nephropathy, presented for emergency renal transplantation. Patient was under hemodialysis with preoperative tests without significant changes, except for increased serum creatinine and urea, compensated metabolic acidosis, mild anemia and diastolic dysfunction at echocardiogram. Spinal morphine $(200 \mu \mathrm{g})$ was proposed aiming at postoperative analgesia. After monitoring with ECG, pulse oximetry and continuous mean blood pressure, patient was sedated with $3 \mathrm{mg}$ midazolam and placed in the sitting position. Spaces between spinous processes $L_{2}-L_{3}$ and $L_{3}-L_{4}$ were easily located. Puncture at $L_{3}-L_{4}$ with $27 \mathrm{G}$ Quincke needle $\left(B\right.$ Braun $\left.{ }^{\circledR}\right)$ was unsuccessful and was followed by attempt at $L_{2}-L_{3}$ when needle deformation was observed. Resistance to mandrel removal was increased and it was mildly deformed (Figure 1). Since there was no CSF reflux we confirmed the suspicion that possible needle progression was due to its deformation and not to its advance toward spinal space. Needle has broken at removal leaving behind a $43 \mathrm{~mm}$ fragment (Figure 2 ) in $\mathrm{L}_{2}$ - $\mathrm{L}_{3}$ interspinous ligament, which was confirmed by fluoroscopy.

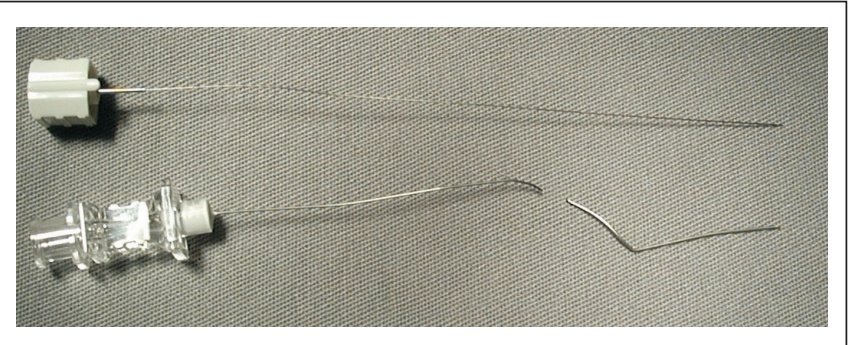

Figure 1 - Fractured Spinal 27G Quincke Needle and its respective Deformed Mandrel

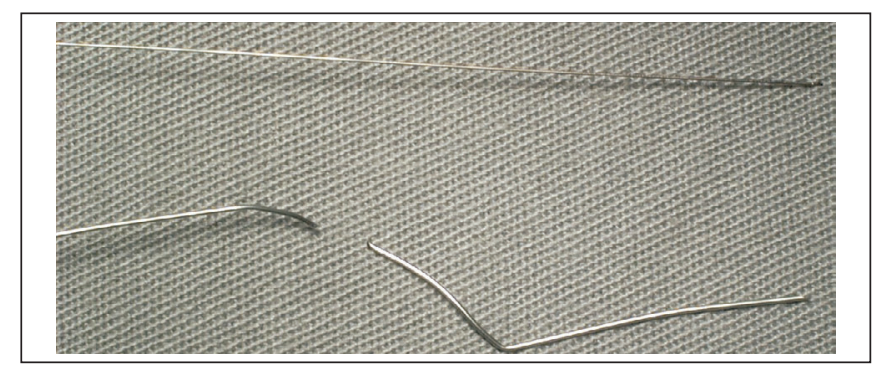

Figure 2 - Detail of spinal 27G Quincke Needle (B Braun ${ }^{\circledR}$ ) Fractured at $43 \mathrm{~mm}$ of its tip and its Mandrel. Removed distal fragment after surgical exploration of Interspinous Ligament $L_{2}-L_{3}$ Region 
Figure 3 shows possible deformation and rupture mechanism. General anesthesia was induced and fragment was removed by fluoroscopic surgical exploration. Renal transplantation was then performed uneventfully.

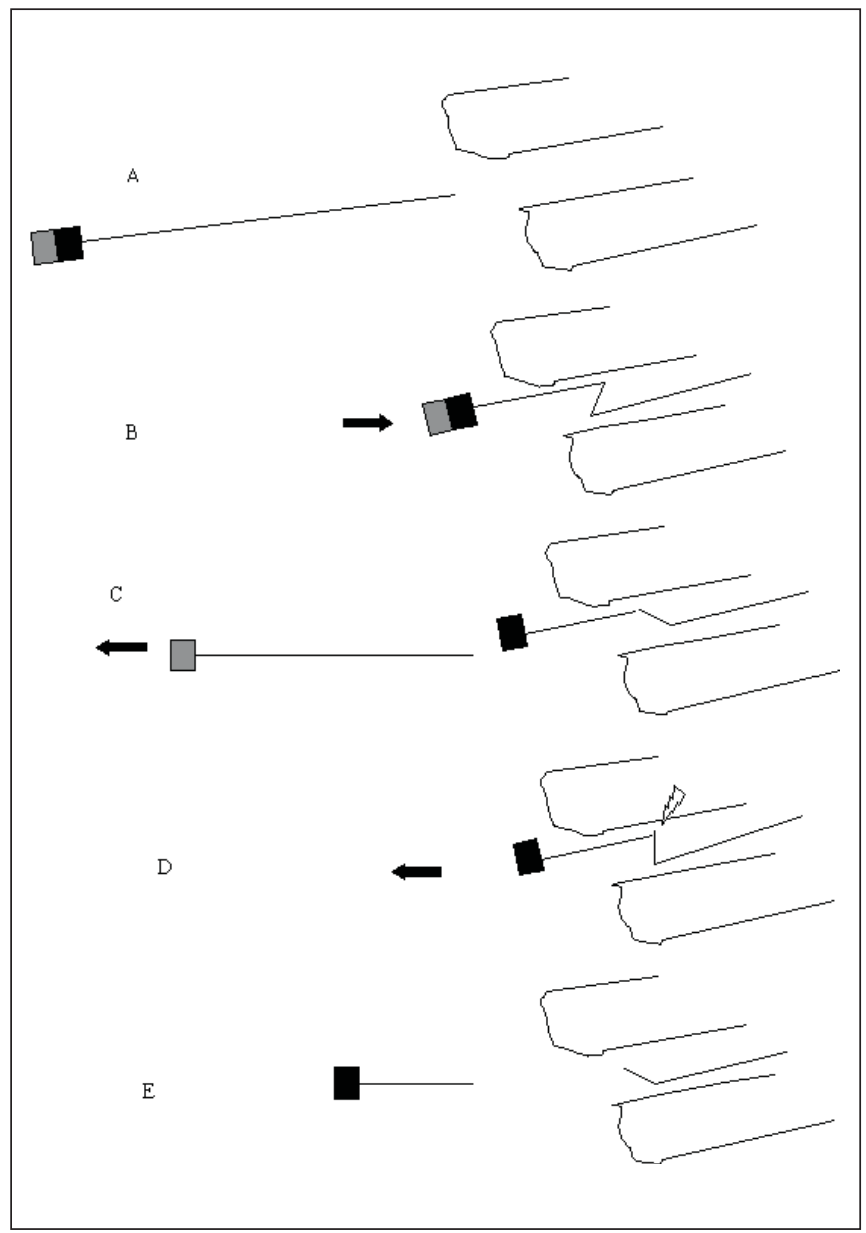

Figure 3 - Drawing showing Possible Needle Deformation (A, B and $\mathrm{C})$ and Fracture (D and E) Mechanisms

\section{DISCUSSION}

Although uncommon and initially surprising, a more careful thought has shown that this complication is not totally unexpected. Benham ${ }^{16}$ has examined 222 needles and has found deformation in $36(16.2 \%)$. Among different needle types and gauges, $25 \mathrm{G}$ needles were significantly less damaged as compared to finer needles. Decreased needle size, with the advantage of decreasing post dural puncture headache, has also made them fragile, leading to higher puncture difficulties and possibility of failures ${ }^{3-9}$. When ligaments are more resistant and puncture is attempted more than once, there may be material stress which may lead to fracture. Oliveira Filho et al. ${ }^{17}$ have found $7.29 \%$ more than four neuraxial blockade attempts. Our patient presented three out of the four predicting factors for difficult puncture studied by this author: age above 40 years, body mass index above $25 \mathrm{~kg} \cdot \mathrm{m}^{2}$ and low height.
Patient did not seem to have anatomic vertebral column changes.

Chaney ${ }^{18}$ calls the attention to the possibility of major deformities in fine needles, in spite of the external appearance of routine puncture. Imbelloni et al. ${ }^{19}$ has reported $29 \mathrm{G}$ needle deformity highlighting that this has never happened with $27 \mathrm{G}$ needles, differently from our case. Although uncommon, there may be marked needle deformation in cases of difficult puncture. Its prompt replacement is recommended for all cases.

The indication of fine needle puncture for our patient is arguable since surgery would be major, he was not a young patient and had predicting factors for difficult puncture. Maybe a thicker 25G needle would not represent increased risk for headache with less chance for deformation. Another point to consider was the insistence in puncturing with $27 \mathrm{G}$ needle. Even without visible signs of needle wear, maybe the best decision would be to replace it after the first unsuccessful attempt.

In conclusion, our case suggests that when there is increased resistance to needle progression or puncture difficulty, the risk for deformation and consequent possibility of fracture should be balanced against fine needle advantages.

\section{REFERÊNCIAS - REFERENCES}

01. Imbelloni LE, Fortis EF - Agulhas, Cateteres, Técnicas e Drogas, em: Imbelloni LE - Tratado de Anestesia Raquidiana. Curitiba, 2001;57-66.

02. Imbelloni LE - O Uso Racional da Raquianestesia, em: Imbelloni LE - Tratado de Anestesia Raquidiana. Curitiba, 2001;74-86.

03. Fortuna A, Fortuna A - Complicações Neurológicas da Raquianestesia, em: Imbelloni LE - Tratado de Anestesia Raquidiana. Curitiba, 2001;164-177.

04. Lambert DH - Complications of spinal anesthesia. Int Anesthesiol Clin, 1989;27:51-55.

05. Bouchacourt V - Falhas na Raquianestesia, em: Imbelloni LE Tratado de Anestesia Raquidiana. Curitiba, 2001;192-198.

06. Liu SS, McDonald S - Current issues in spinal anesthesia. Anesthesiology, 2001;94:888-906.

07. Casati A, Vinciguerra F - Intrathecal anesthesia. Curr Opin Anesthesiol, 2002;15:543-551.

08. Horlocker TT, McGregor DG, Matsushige DK et al - A retrospective review of 4767 consecutive spinal anesthetics: central nervous system complications. Perioperative Outcomes Group. Anesth Analg, 1997;84:578-584.

09. Ganem EM, Castiglia YMM, Vianna PTG - Complicações neurológicas determinadas pela anestesia subaracnóidea. Rev Bras Anestesiol, 2002;52:471-480.

10. Auroy Y, Narchi P, Messiah A et al - Serious complications related to regional anesthesia: results of a prospective survey in France. Anesthesiology, 1997;87:479-486.

11. Faccenda KA, Finucane BT - Complications of regional anaesthesia. Incidence and prevention. Drug Saf, 2001;24:413-442.

12. Ben-David B, Rawa R - Complications of neuroaxial blockade. Anesthesiol Clin North America, 2002;20:669-693.

13. Hemmerling TM, Babin D - Phonomyography-acoustic myography using condenser microphones: a promising new method of monitoring neuromuscular transmission. Anaesth Intensive Care, 2002; 30: 532-533. 
14. Crowhurst JA - Fractured 27 gauge Whitacre spinal needle. Anaesth Intensive Care, 1997;25:317-318.

15. Crowhurst JA, Plaat F - Fractured fine gauge narrow, atraumatic (pencil point) spinal needles. Anaesth Intensive Care, 1998;26:457-458.

16. Benham M - Spinal needle damage during routine clinical practice. Anaesthesia, 1996;51:843-845.

17. de Filho GR, Gomes HP, da Fonseca MH et al - Predictors of successful neuroaxial block: a prospective study. Eur J Anesthesiol, 2002;19: 447-451.

18. Chaney MA, Brey SJ - Severe deformation of small-gauge spinal needle. Anesth Analg, 1993;77:401-402.

19. Imbelloni LE, Sobral MGV, Carneiro ANG - Raquianestesia com agulhas finas tipo Quincke. Rev Bras Anestesiol, 1994;44: 293-294.

\section{RESUMEN}

Cruvinel MGC, Andrade AVC - Fractura de Aguja durante Punción Subaracnóidea. Relato de Caso

JUSTIFICATIVA Y OBJETIVOS: Después de la introducción de las agujas de calibre fino (26G, 27G y 29G) y la consecuente disminución de la incidencia de cefalea pos-punción de dura-máter, la raquianestesia viene siendo cada día más empleada. Sus complicaciones son poco frecuentes. Recientemente, sin embargo, fue observada una complicación aparentemente rara, de fractura de la aguja durante punción subaracnóidea. El objetivo de este relato es registrar el hecho y exponer las posibles causas y su prevención.

RELATO DEL CASO: Paciente del sexo masculino, 53 años, $90 \mathrm{~kg}, 175 \mathrm{~cm}$, portador de hipertensión arterial sistémica crónica e insuficiencia renal crónica se presentó, en carácter de urgencia, para trasplante renal. Fue propuesta la administración de morfina subaracnóidea con la finalidad de analgesia pós-operatoria. Inicialmente se intentó, sin éxito, punción en $L_{3}-L_{4}$ con aguja $27 \mathrm{G}$, se siguió una tentativa de punción en $L_{2}-L_{3}$, cuando se percibió deformación de la aguja. Al ser retirada la aguja se partió, permaneciendo un fragmento de $43 \mathrm{~mm}$ en el ligamento interespinoso de $L_{2}-L_{3}$, confirmado por fluoroscopia. Fue inducida anestesia general y se procedió a una exploración quirúrgica guiada por fluoroscopia con retirada del fragmento de la aguja. A continuación, el trasplante renal fue realizado sin intercurrencias.

CONCLUSIONES: La disminución del calibre de las agujas, que trajo la ventaja de la reducción de la incidencia de cefalea después de punción de la dura-máter, hizo también con que ellas se volviesen más débiles. En casos donde los ligamentos son más fuertes y la punción intentada más de una vez, puede haber deformación del material que se pone quisquilloso a la quiebra. Se concluye que en situaciones en las que hay resistencia aumentada por el pasaje de la aguja o dificultad de punción, el riesgo de su deformación y la posibilidad de fractura deben ser afrontados con las ventajas del uso de agujas de fino calibre. 